\title{
Comparison of Methods for Tool Localization in Biological Tissue from 3D Ultrasound Data
}

\author{
Martin Barva ${ }^{1,2}$, Jan Kybic ${ }^{1}$, Václav Hlaváč ${ }^{1}$ \\ ${ }^{1}$ Center for Machine Perception \\ Czech Technical University (CTU) \\ Prague, Czech Republic \\ Email: barvam1@cmp.felk.cvut.cz
}

\author{
Hervé Liebgott ${ }^{2}$, Christian Cachard $^{2}$ \\ ${ }^{2}$ CREATIS \\ Université Claude Bernard Lyon I (UCB) \\ CNRS UMR 5515, U 630 INSERM \\ Lyon, France
}

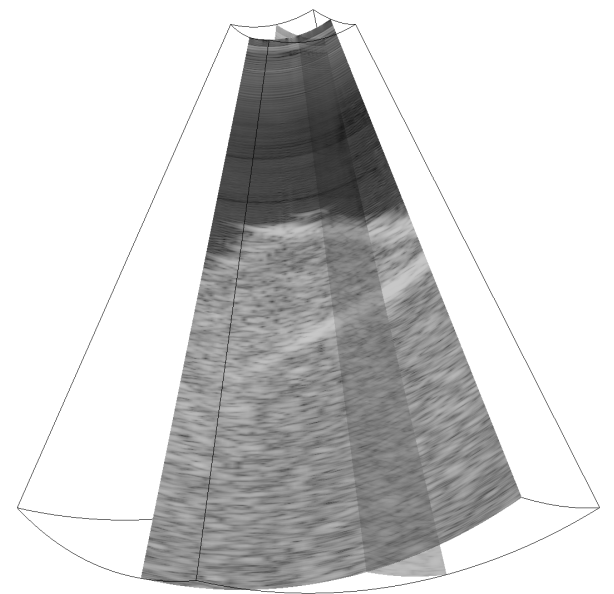

\begin{abstract}
In medical applications, miniature surgical instruments such as needles, or electrodes are introduced into human body. The position of instrument in tissue can be estimated using 3D ultrasound. In previous publications, we introduced two novel algorithms for automatic electrode localization from 3D ultrasound images. The first method is based on the Parallel Integral Projection (PIP) transform, a modification of the Radon transform. We showed that the axis of the electrode can be estimated from the maximum of PIP transformation. To accelerate search for the maximum, a hierarchical mesh-grid algorithm is implemented. In second method, the electrode axis is described by a cubic polynomial. The distribution of voxel intensities inside the electrode region is a priori estimated from acquired data. The model parameters are robustly estimated using the RANSAC estimator. In this paper, their performance in terms of accuracy is compared. A series of tests on numerical phantoms created with the FIELD II simulation program were performed to quantitatively evaluate the localization accuracy. We observed a decrease in accuracy when artificial noise was added to the input data. The algorithms were also tested on real ultrasound data of a cryogel phantom comprising metallic electrode.
\end{abstract}

\section{INTRODUCTION}

It is often necessary to know the position of surgical tool in real time. For example, in biopsy, tissue samples are extracted from a particular region by means of a thin needle [1]. In breast cancer therapy, radioactive substance is injected near the tumor [2]. In the field of neurobiological research, there are experiments, where the electrode activity of a small group of neurons is recorded through a thin tungsten electrode [3]. Precise navigation of surgical instruments is vital in intracranial surgery, where there is a risk of damaging major blood vessel.

The navigation of surgical tools is based on visual information provided by medical imaging modality such as the ultrasound, magnetic resonance, or computed tomography. The localization accuracy of a surgical tool inside tissue is improved if the tool position is determined automatically. Another application of automatic tool localization is in the domain of visualization of acquired data. In intraoperative procedures, 3D imaging modalities are often employed to obtain complex 3D images of scanned region. Using the tool position, we can easily determine a planar section comprising the tool and display it on a computer screen.
The ultrasound modality has several advantages over other imaging modalities such as 3D near real-time imaging capabilities, non-invasiveness, no ionizing radiation, compatibility with metallic surgical instruments, portability, affordability. Fig. 1 depicts an example of a 3D ultrasound image of a polyvinyl alcohol (PVA) cryogel phantom [4] containing an electrode in water. In publications [5], [6] we presented two algorithms that permit to automatically determine the electrode position from 3D ultrasound images. First method is based on maximizing the parallel projection of input 3D image. A thin electrode may bend slightly while introduced into tissue. To localize electrode with a curvilinear axis, a second approach based on model fitting was developed and implemented. It describes the electrode with a model whose parameters are estimated using the RANSAC algorithm. Here we briefly review both methods and evaluate their performance on simulated and real ultrasound data in term of accuracy and robustness to image quality. 


\section{PROPOSED METHODS}

The input to both algorithms is a $3 \mathrm{D}$ image that is described by an image function

$$
\mathcal{I}: \mathbf{A} \rightarrow \mathbf{B}
$$

where $\mathbf{A} \subset \mathbb{R}^{3}$ is a set of voxels coordinates and $\mathbf{B} \subset \mathbb{R}_{0}^{+}$is a set of non-negative voxel intensities.

We assume that first, the electrode shape is cylindrical with the length much greater than its diameter. Second, we assume that electrode voxel intensities are superior to the background voxels intensities.

\section{A. Method I - Maximizing Parallel Projection}

The electrode axis is found by maximizing the Parallel Integral Projection transformation. The Parallel Integral Projection is a transform that maps an image function $\mathcal{I}: \mathbf{A} \rightarrow \mathbf{B}$ to a function $\mathcal{P}_{\mathcal{I}}: \mathbb{R}^{4} \rightarrow \mathbb{R}$ describing its projections as a function of the $2 \mathrm{D}$ displacements $(u, v)$ and the projection direction determined by two angles $(\alpha, \beta)$. Formally, the PIP transformation $\mathcal{P}_{\mathcal{I}}$ of $\mathcal{I}$ is given by

$$
\mathcal{P}_{\mathcal{I}}(u, v, \alpha, \beta)=\int_{-\infty}^{\infty} \mathcal{I}\left(\mathbf{R}(\alpha, \beta) \cdot(u, v, \tau)^{T}\right) \mathrm{d} \tau,
$$

where $\mathbf{R}(\alpha, \beta)$ is the rotation matrix representing a rotation around the $x$-axis by angle $\alpha$, and around the $y$-axis by angle $\beta$. The value $\mathcal{P}_{\mathcal{I}}(u, v, \alpha, \beta)$ is equal to the integral of the function $\mathcal{I}$ along a line of integration defined by $\mathbf{R}(\alpha, \beta)$. $(u, v, \tau)^{T} ; \tau \in \mathbb{R}$. The PIP transformation is periodical along the parameters $\alpha, \beta$ with period $\pi$.

If a 3D image contains a negligibly thin electrode, the PIP transformation $\mathcal{P}_{\mathcal{I}}$ has maximum when the line of integration coincides with the electrode axis [5]. Let $\left(u_{\max }, v_{\max }, \alpha_{\max }, \beta_{\max }\right)$ be the point, where $\mathcal{P}_{\mathcal{I}}$ reaches the maximum. Then the analytic equation of the electrode axis $l(t)$ is given by

$$
l(t)=\mathbf{R}\left(\alpha_{\max }, \beta_{\max }\right) \cdot\left(\begin{array}{c}
u_{\max } \\
v_{\max } \\
t
\end{array}\right) ; \quad \forall t \in \mathbb{R} .
$$

The maximum of $\mathcal{P}_{\mathcal{I}}$ is sought on a discrete grid. In order to accelerate the search, the discretization along the dimensions $\alpha, \beta$ is done using the hierarchical mesh-grid method [7]. It is controlled through parameters $\Delta_{\alpha}^{1}, \Delta_{\beta}^{1}$, resp. $\Delta_{\alpha}^{N}, \Delta_{\beta}^{N}$ which are the initial, resp. final discretization steps of $\alpha, \beta$. The discretization steps $\Delta_{u}, \Delta_{v}$ along the dimensions $u, v$ are kept constant, since the spatial density of integration lines must be inferior to electrode diameter.

\section{B. Method II - Model fitting with RANSAC}

The method starts by pre-segmentation of voxels from the set $\mathbf{A}$ in two disjoined sets $\mathbf{A}_{e}$ and $\mathbf{A}_{b}$. This is done by thresholding with a threshold set to such a value that $\mathbf{A}_{e}$ consists of $10 \%$ of voxels with the highest intensities. This operation roughly distinguishes between the electrode voxels and background voxels and accelerates the algorithm since only the voxels from $\mathbf{A}_{e}$ are processed in consecutive computations.
1) Electrode model: The electrode axis is modeled by a cubic polynomial $l_{\Theta}(t)$ given by

$$
\begin{aligned}
l_{\Theta}(t): x(t) & =a_{0}+a_{1} t+a_{2} t^{2}+a_{3} t^{3} \\
y(t) & =b_{0}+b_{1} t+b_{2} t^{2}+b_{3} t^{3} \\
z(t) & =c_{0}+c_{1} t+c_{2} t^{2}+c_{3} t^{3}, \quad t \in \mathbb{R},
\end{aligned}
$$

where $\Theta=\left(a_{0}, a_{1}, a_{2}, a_{3}, b_{0}, b_{1}, b_{2}, b_{3}, c_{0}, c_{1}, c_{2}, c_{3}\right)$ is the parameter vector determining the curve position and shape.

The second part of the model describes the electrode voxel intensities with respect to the distance from the axis. We a priori estimated a joint probability density function $p(v, d)$ between voxel intensity $v$ and the voxel-to-axis distance $d$. The expected value of the intensity at distance ? is

$$
h(d)=E[v \mid d]=\int_{-\infty}^{\infty} v p(v, d) \mathrm{d} v .
$$

2) Estimating parameter vector $\Theta$ : The parameter vector $\hat{\Theta}$ such that

$$
\hat{\Theta}=\arg \min _{\Theta} C(\Theta)
$$

where $C(\Theta)$ is a model quality evaluating cost function (7), is estimated by the RANSAC algorithm [8]. In each iteration $i$, a random subset $\mathbf{D}_{i}$ of four points is selected from all electrode voxels $\mathbf{A}_{e}$. A parameter vector $\Theta_{i}$ is calculated to fit the cubic polynomial to the subset $\mathbf{D}_{i}$. The quality of the model is determined using a cost function given by

$$
C\left(\Theta_{i}\right)=\sum_{\mathbf{x} \in A_{e}}\left[I(\mathbf{x})-h\left(d\left(l_{\Theta_{i}}(t), \mathbf{x}\right)\right)\right]^{2}
$$

where $d\left(l_{\Theta_{i}}(t), \mathbf{x}\right)$ is the distance of the point $\mathbf{x}$ from the curve $l_{\Theta_{i}}(t), I(\mathbf{x})$ is the true intensity and $h\left(d\left(l_{\Theta_{i}}(t), \mathbf{x}\right)\right)$ is expected voxel intensity. The best model in the sense of $C(\Theta)$ over all iterations is selected. The number of iterations is set to such a value that the probability of the event "better-thancurrently-best estimated model is missed" is inferior to some predetermined constant $\eta$.

\section{EXPERIMENTS}

Two types of 3D ultrasound images were used to test the methods: (i) simulated phantoms to evaluate the algorithm accuracy, (ii) real ultrasound data of PVA cryogel phantom containing an electrode were used to test its performance in realistic conditions.

\section{A. Accuracy assessment}

In following text the localization accuracy is quantified by a measure $\varepsilon_{\text {axis }}$ given by

$$
\varepsilon_{\text {axis }}=\max \left\{\left\|E_{1}-Q_{1}\right\|,\left\|E_{2}-Q_{2}\right\|\right\}
$$

where $E_{1}, E_{2}$ are the ground-truth coordinates of electrode endpoints and $Q_{1}, Q_{2}$ are the intersections of estimated axis $l(t)$ with normals to the electrode axis through $E_{1}, E_{2}$.

\section{B. Method parameter settings}

In the experiments described in this section, we used following algorithm parameter settings. 
1) Method I: $\Delta_{\alpha}^{1}=\Delta_{\beta}^{1}=32^{\circ}, \Delta_{\alpha}^{N}=\Delta_{\beta}^{N}=1^{\circ}$ and $\Delta_{v}=\Delta_{v}=1 \mathrm{~mm}$. These parameters were deduced from a priori known diameter and length of sought electrode [5].

2) Method II: The number of iterations in RANSAC estimator was set to 500, which corresponds to $\eta$ of 0.05 .

\section{Numerical phantom}

Numerical phantoms were generated using the ultrasound simulator FIELD II [9]. A set of MATLAB functions permit to simulate realistic 3D ultrasound images.

The parameters of the simulator were set to imitate the ultrasound scanner Kretz Voluson 530D (Kretztechnik AG, Austria). We used a multi-element transducer to produce a 2D sector scan plane, which was tilted to scan a 3D region. The sector scan plane of angle $40^{\circ}$ was composed of 71 beams and 53 scan planes were acquired with a tilt angle of $40^{\circ}$.

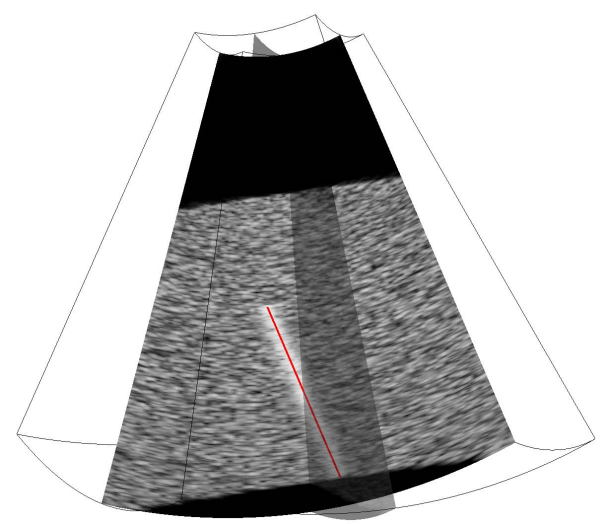

Fig. 2. Simulated 3D ultrasound image using the FIELD II simulator. The red line segment marks axis found with Method I.

We simulated a series of numerical phantoms representing a cuboid region of biological tissue comprising a highly reflecting inclusion such a metallic electrode. The dimensions

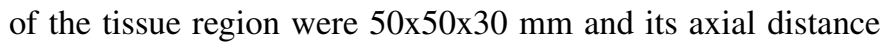
from the probe was set to $35 \mathrm{~mm}$. To approximate the speckle pattern of biological tissue, the tissue diffuser spatial density was 3 per $\mathrm{mm}^{3}$. Their reflection coefficients had normal distribution with zero mean and variance 1 . The electrode inside tissue was represented by a cylindrical region of 0.3 $\mathrm{mm}$ in diameter containing diffusers with spatial density 125 per $\mathrm{mm}^{3}$. Their reflection coefficients were constant and equal to 5. Fig. 2 depicts an example of a 3D numerical phantom simulated in FIELD II.

1) Varying background noise: In real applications, the intensity of electrode and background voxels vary. To investigate the influence of background noise, we simulated numerical phantoms with fixed electrode position while increasing the variance of background voxels, e.g. the noise. Given the mean of electrode voxel intensity $\mu_{e l}$ and the mean of background voxel intensity $\mu_{b g}$, we define a parameter SNR given by

$$
\mathrm{SNR}=\frac{\mu_{e l}}{\mu_{b g}}
$$

to quantify the ratio of electrode intensity to background noise.

Fig. 3 shows achieved localization accuracy for Method I and II as a function of SNR. For high SNR, Method I outperforms Method II. However, as the background noise increases ( $\mathrm{SNR} \leq 3.75)$, Method II localizes electrode axis with higher accuracy compared to Method I.

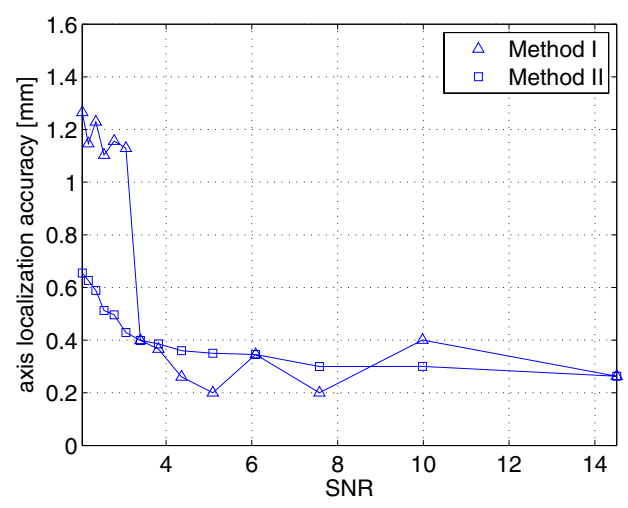

Fig. 3. Axis localization accuracy as a function of signal-to-noise ratio of voxel intensities.

2) Varying electrode orientation: Nine simulated phantoms comprising an electrode in different positions and orientations were simulated. The electrode angle with respect to the probe axis was varied from $0^{\circ}$ to $160^{\circ}$. Localization accuracy for both methods as a function of this angle is depicted in Fig. 4. Notice that in both cases, the localization is the most accurate when the electrode is perpendicular to the probe axis. This is due to different spatial resolution in axial, lateral and azimuthal direction of a ultrasound scanning system. When the electrode is perpendicular to the probe axis, its contours are more clearly defined than for other orientations permitting better localization.

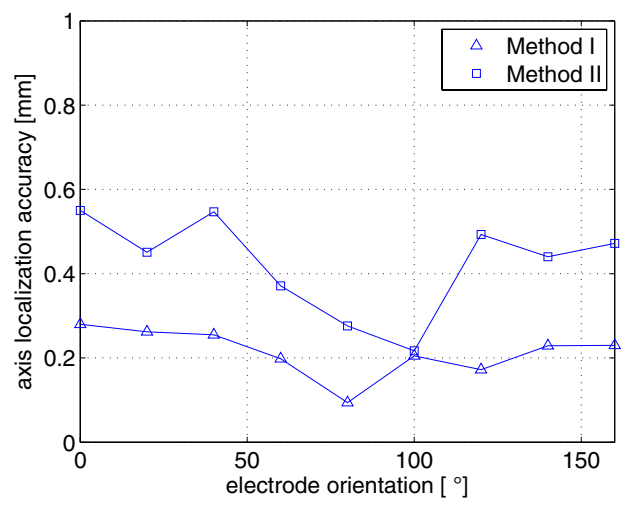

Fig. 4. localization accuracy as a function of the angle between the electrode and probe axis.

\section{Polyvinyl cryogel phantom}

The proposed method was also tested on real 3D ultrasound data. To simulate biological tissue with a highly reflecting 
inclusion, a PVA cryogel phantom of the size 50x50x50 mm was employed. Inside the phantom there was a thin tungsten electrode of $150 \mu \mathrm{m}$ in diameter and length $30 \mathrm{~mm}$. The phantom was scanned with a $3 \mathrm{D}$ ultrasound scanner Voluson 530D equipped with a mechanically tilted transducer operating at central frequency 7.5 MHz. Total scanning (volume) angle was set to $40^{\circ}$ at interval $0.75^{\circ}$. Angle of acquired Bmode sector images was $40^{\circ}$ with the angular step $0.5^{\circ}$. The scanning depth was $6 \mathrm{~cm}$. Acquired data were transfered to a computer where a 3D image of the size 1000x $1000 \times 1500$ voxels was reconstructed (Fig. 5).

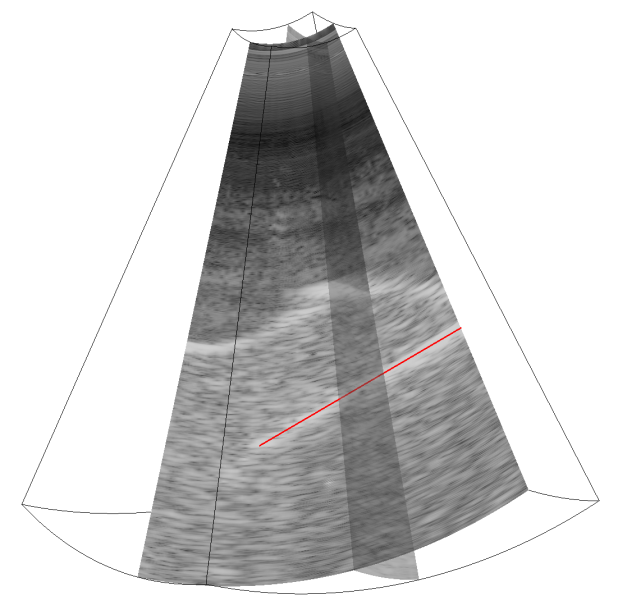

Fig. 5. Example of a PVA cryogel phantom comprising a tungsten electrode. Axis found with Method I is marked in red.

To evaluate the accuracy $\varepsilon_{\text {axis }}$ according to (8), the coordinates of electrode endpoints $E_{1}, E_{2}$ were manually determined in each dataset and considered as ground-truth. Fig. 6 shows the localization accuracy for both methods. The average $\varepsilon_{\text {axis }}$ for Method I was $0.15 \mathrm{~mm}$ and $0.35 \mathrm{~mm}$ for Method II.

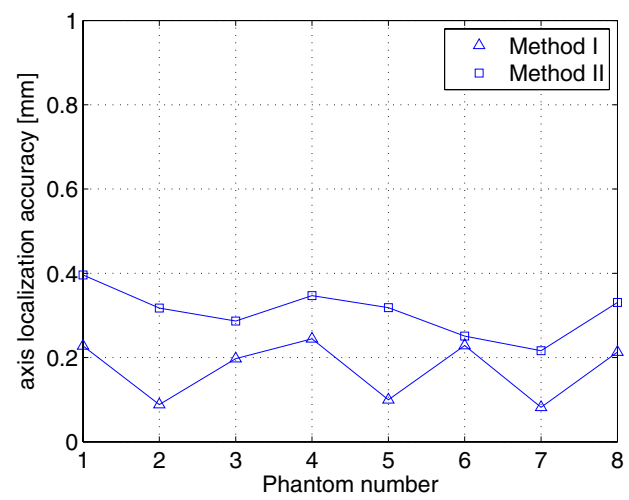

Fig. 6. Axis localization accuracy for a set of acquired 3D ultrasound images of a PVA phantom.

\section{CONCLUSION}

The ultrasound imaging modality is commonly used in intraoperative imaging for the guidance of surgical tools. To facilitate this task, we developed two methods for automatic localization of metallic electrode from 3D ultrasound images. The experiments performed on simulated and real ultrasound images show that the localization accuracy of both methods is on order of hundreds $\mu m$ regardless the electrode position and orientation. The method based on maximizing the parallel projection outperforms in accuracy the model fitting algorithm. However, for images with poor signal-to-noise ratio the model fitting approach is more accurate and its low computational time permits real-time performance.

\section{ACKNOWLEDGEMENT}

The 1st author was partially supported by The Grant Agency of the Czech Academy of Sciences under project 1ET101050403, by the Internal Grant of the Czech Technical University in Prague and by the French Embassy under the project of doctoral studies with co-supervision. The 2nd author was supported by the The Czech Ministry of Education under project MSM6840770012.

\section{REFERENCES}

[1] A. Abati and A. Simsir. Breast fine needle aspiration biopsy: prevailing recommendations and contemporary practices. Clinics in laboratory medicine, 25(4):631-654, December 2005.

[2] K. Kern. Sentinel lymph node mapping in breast cancer using subareolar injection of blue dye. Journal of the American College of Surgeons, 189(6):539-545, December 1999.

[3] R. L. Alterman, D. Sterio, A. Beric, and P. J. Kelly. Microelectrode recording during posteroventral pallidotomy: Impact on target selection and complications. Neurosurgery, 44(2):315-321, February 1999.

[4] K. C. Chu, , and B. K. Rutt. Polyvinyl alcohol cryogel: An ideal phantom material for $\mathrm{mr}$ studies of arterial flow and elasticity. Magnetic Resonance in Medecine, 37(2):314-319, 1997.

[5] Martin Barva, Jan Kybic, Jean-Martial Mari, and Christian Cachard. Radial Radon transform dedicated to micro-object localization from radio frequency ultrasound signal. In Marjorie Passini Yuhas, editor, IEEE UFFC: Proceedings of the IEEE International Frequency Control Symposium and Exposition, pages 1836-1839, 445 Hoes Lane, Piscataway, USA, August 2004. IEEE, IEEE Operations Center.

[6] Martin Barva, Jan Kybic, Jean-Martial Mari, Christian Cachard, and Václav Hlaváč. Automatic localization of curvilinear object in 3D ultrasound images. SPIE International Symposium on Medical Imaging, February 2005.

[7] E. Heitz, P. Perez, and P. Bouthemy. Multiscale minimization of global energy functions in some visual recovery problems. Computer Vision, Graphics, and Image Processing, 59(1):125-134, 1994.

[8] M. A. Fischler and R. C. Bolles. Random sample consensus: A paradigm for model fitting with applications to image analysis and automated cartography. CACM, 24(6):381-395, June 1981.

[9] J. A. Jensen. Field: A program for simulating ultrasound systems. In Medical and Biological Engineering and Computing, volume 34, pages 351-353, 1996. 